\title{
MODELLI TEORICI DI FEDERALISMO
}

\author{
di Daniela Giannetti
}

\section{Introduzione}

Tutti gli studiosi concordano nel considerare l'unione degli stati americani creata con la Costituzione del 1787 il primo esempio di federalismo in senso moderno. Qual è la novità storica del federalismo americano? In sintesi, si può affermare che essa consiste in un rafforzamento del potere centrale che si accompagna a un insieme di garanzie costituzionali circa le sfere di autorità o la ripartizione di funzioni tra differenti livelli di governo. La Costituzione di Filadelfia viene inoltre generalmente considerata un esempio di deliberata progettazione istituzionale e i vari saggi che compongono il Federalist - scritti da Hamilton, Jay e Madison allo scopo di illustrare i vantaggi che sarebbero derivati da un'organizzazione federale degli stati indipendenti allora uniti nella confederazione nordamericana sono considerati la prima articolazione compiuta della teoria federalista. Dall' «invenzione» del sistema americano, che introduce un'innovazione sostanziale nella storia delle istituzioni occidentali, poi ampiamente imitata in numerosi paesi, il federalismo è diventato una delle realtà politiche più importanti e uno dei temi più dibattuti nella letteratura ${ }^{1}$.

' La vastissima letteratura sull'argomento spazia dalla storia del pensiero politico, anche anteriore alla creazione degli Stati Uniti d'America, all'analisi comparata di un insieme vasto e differenziato di sistemi politici - dal Canada alla Germania, dall'Australia alla Svizzera - e alle loro trasformazioni nel tempo, nonché all'azione dei movimenti federalisti (non presa in considerazione in questo lavoro). Per il primo aspetto si veda, ad esempio, la letteratura citata in Albertini (1979) e i numerosi articoli comparsi su «Publius. The Journal of Federalism», la rivista edita da Daniel J. Elazar, uno degli studiosi più noti della materia; questa stessa rivista è utile per l'analisi di particolari aspetti o paesi, ma da questo punto di vista soltanto l'evoluzione del sistema americano richiederebbe una bibliografia specifica (si rimanda perciò alle altre indicazioni bibliografiche

RIVISTA ITALIANA DI SCIENZA POLITICA / a. XXV, n. 2, agosto 1995 
Le teorie delle istituzioni si possono sommariamente suddividere in due gruppi: quelle che sottolineano gli aspetti evolutivi, o di emergenza spontanea e di adattamento funzionale, e quelle che mettono in luce le intenzioni e le motivazioni degli attori rilevanti posti in un contesto di interazione strategica (Knight 1992). Gli autori del Federalist possono essere considerati a pieno titolo esponenti - o meglio precursori - del secondo gruppo di teorie, dal momento che assumono che gli attori politici siano motivati dall'autointeresse e analizzano il comportamento individuale come prodotto degli incentivi istituzionali ${ }^{2}$. L'approccio basato sulla razionalità degli attori sembra quindi particolarmente pertinente per analizzare il federalismo, non solo perché molti contributi importanti - Buchanan, Riker, Ostrom - si richiamano ad essa. Anche un noto studioso del federalismo come Elazar (1987), che pure non appartiene a questa tradizione di ricerca, ha affermato:

l'idea federale stessa si fonda sul principio che le istituzioni e le relazioni sociali e politiche si stabiliscono meglio attraverso patti, contratti o altri ordinamenti costituzionali, invece che attraverso il semplice sviluppo organico (o in aggiunta ad esso); in altre parole, che gli uomini sono in grado di scegliere le costituzioni (Elazar 1987, 33).

All'interno dell'approccio razionale si possono distinguere varie prospettive o programmi di ricerca. La prospettiva economica ha prevalentemente analizzato il federalismo in una accezione ristretta, come federalismo fiscale, e in chiave normativa, con l'obiettivo cioè di prescrivere la «dimensione ottima delle giurisdizioni» o l'assegnazione ottimale delle funzioni tra differenti livelli di governo. Si tratta di un contributo importante perché, in una fase segnata dallo «scetticismo comportamentista in merito agli effetti delle istituzioni» (Beam, Conlan e Walker 1983, 259), la teoria economica ha fornito elementi probatori all'ipotesi secondo cui l'organizzazione territoriale del governo comporta effetti significativi sulle politiche pubbliche e sulla re-

contenute nel presente lavoro). Il volume di Brosio (1994) rappresenta un contributo molto utile all'analisi comparata dei sistemi federali; si veda anche Elazar (1993) per una sintetica rassegna.

${ }^{2}$ Cfr. The Federalist, ed. or. 1788, varie edizioni; trad. it. Pisa, Nistri Lischi, 1955. Per una ricostruzione analitica del pensiero dei padri fondatori della Costituzione americana molto importante è il volume di Ostrom (1987); si vedano anche i saggi raccolti in Gwartney e Wagner (1988) e in Grofman e Wittman (1989). Un richiamo ai Founding Fathers è contenuto anche in numerosi lavori di Buchanan. 
sponsiveness del governo alle preferenze dei cittadini. Uno dei limiti di questa prospettiva, esplicitamente riconosciuto, è che essa non giustifica il federalismo, ma il decentramento politico (Oates 1972, 17-18). In altre parole, essa ignora le caratteristiche istituzionali che distinguono il federalismo rispetto a un qualunque assetto decentrato. Un passo avanti in questa direzione è compiuto dalla public choice, che rimanda a una vasta letteratura generata dal pionieristico lavoro di Buchanan e Tullock (1962).

Nella prospettiva della razionalità degli attori si è sviluppata anche un'analisi strettamente politologica del federalismo. In contrapposizione con i modelli economici, Riker sottolinea in primo luogo le caratteristiche del federalismo come assetto istituzionale distinto dal decentramento. In secondo luogo, propone una teoria della genesi e della stabilità delle istituzioni federali articolata in numerosi lavori (Riker 1964, 1975 e 1987).

All'analisi del problema della stabilità istituzionale offre un contributo importante la teoria politica positiva, che costituisce una parte sostanziale del programma di ricerca noto come neoistituzionalismo ${ }^{3}$. Quest'ultimo si caratterizza per l'uso di strumenti analitici derivati dalla microeconomia, dall'economia dei costi di transazione, dalla teoria dei giochi e dalla teoria della scelta sociale per analizzare il problema della genesi delle istituzioni e degli effetti di strutture e procedure sugli esiti delle scelte collettive. Originariamente caratterizzata da una concentrazione pressoché esclusiva sulle regole di voto, la teoria politica positiva si è recentemente rivolta all'analisi di strutture istituzionali più complesse, quali il bicameralismo, la separazione dei poteri, il federalismo. La necessità di una maggiore integrazione tra le diverse prospettive viene sottolineata da vari autori (Hamlin 1985; Wiseman 1990).

Questo lavoro non pretende di costituire una rassegna completa, ma si propone di illustrare - nell'ottica della scienza politica - $\mathrm{i}$ contributi più importanti all'analisi del federalismo entro i vari filoni della letteratura che ha in comune l'adesione all'individualismo metodologico e l'assunzione di razionalità degli attori. L'esposizione sarà organizzata intorno ai seguenti temi (oggetto dei relativi paragrafi): a) il federalismo nell'approccio

\footnotetext{
${ }^{3}$ Per un'introduzione al neoistituzionalismo rimando a Giannetti (1993) e alla letteratura ivi citata.
} 
economico e nella public choice; b) la teoria di Riker e la «legge delle origini federali»; $c$ ) il federalismo nella teoria politica positiva. Anche se il taglio del lavoro sarà prevalentemente teorico, si farà riferimento ai casi empirici quando ciò si renderà necessario per illustrare i passaggi dell'argomentazione, rimandando alle citazioni in nota per gli approfondimenti relativi a singoli sistemi politici.

\section{Il federalismo nell'approccio economico e nella public choice}

È noto che la teoria economica offre una interpretazione stilizzata dell'insorgenza dello stato e delle istituzioni politiche. Secondo questa interpretazione, la creazione dello stato (o del governo) nelle comunità umane rappresenta una risposta a quei problemi che non trovano soluzione nel mercato, quali la produzione di beni pubblici o l'eliminazione di «esternalità» negative. Applicata all'analisi del federalismo, questa interpretazione genera le seguenti domande: perché esistono gli stati decentrati, e qual è il grado di decentramento ottimale? La risposta risiede essenzialmente in considerazioni di efficienza.

Punto di partenza è l'idea che il governo svolga tre principali funzioni economiche: quella allocativa (relativa alla produzione di beni e servizi) quella distributiva (la distribuzione del reddito) e quella di stabilizzazione (l'uso di strumenti fiscali e monetari per aumentare la produzione e l'occupazione mantenendo stabili i prezzi). Mentre la funzione distributiva e quella di stabilizzazione sono tipicamente attribuite al governo centrale, la responsabilità della funzione allocativa è condivisa con i livelli di governo inferiori (Musgrave 1959; Oates 1972). La possibilità di attribuire la funzione allocativa a livelli di governo diversi da quello centrale dipende dalle proprietà spaziali dei beni pubblici, in particolare dall'esistenza di beni pubblici loca$l i$, i benefici dei quali ricadono su un ambito geografico limitato. A differenza dei beni pubblici puri (ad esempio, la difesa nazionale) i beni pubblici locali sono prodotti in modo più efficiente a livelli di governo inferiore. Poiché i cittadini hanno preferenze diverse in merito al livello di produzione dei beni pubblici, la capacità dei governi decentrati di diversificare il loro output in accordo con le preferenze locali migliora l'allocazione delle risorse. I guadagni di efficienza del decentramento sono illustrati nella figura 1 . 


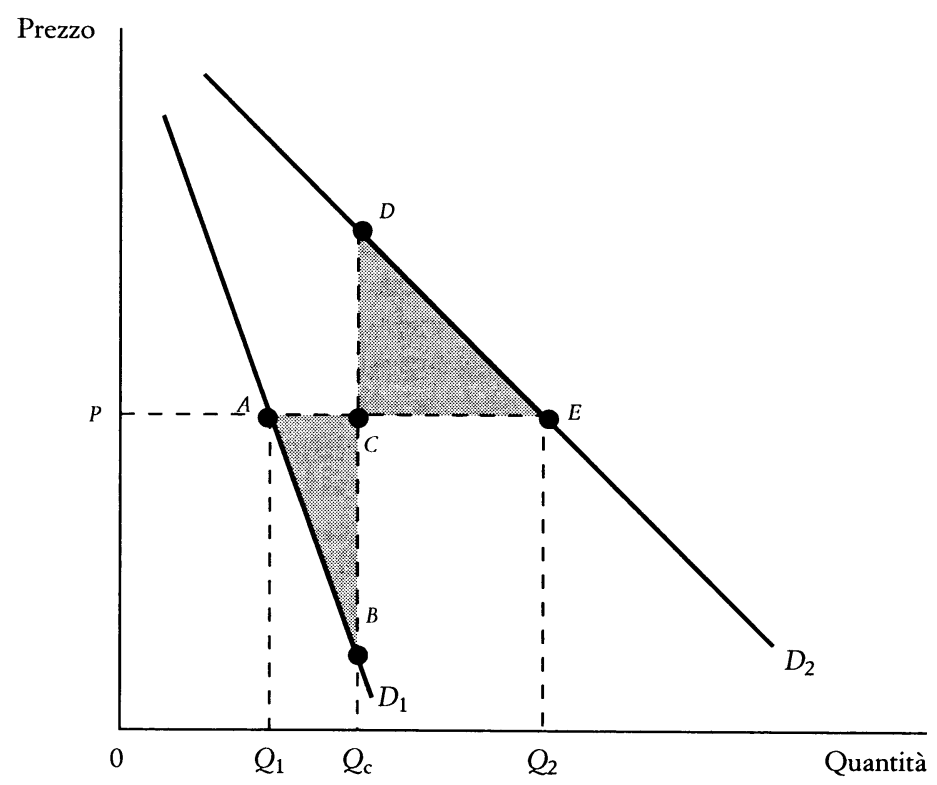

FIG. 1. I guadagni di efficienza del decentramento.

Fonte: Brown e Jackson (1990).

Supponiamo che la popolazione sia divisa in due gruppi. Assumiamo che gli individui all'interno dei diversi gruppi abbiano preferenze omogenee ma che le curve di domanda tra $\mathrm{i}$ due gruppi differiscano. Ad esempio, tutti gli individui del gruppo 1 possiedono la curva di domanda $D_{1}$ e tutti gli individui del gruppo 2 la curva di domanda $\mathrm{D}_{2}$. Assumiamo anche che il servizio pubblico sia fornito a un costo costante per ciascuno. Il livello preferito di output per i membri del gruppo 1 è $\mathrm{Q}_{1}$ e per i membri del gruppo 2 è $\mathrm{Q}_{2}$. In un sistema centralizzato sarebbe fornito un livello uniforme di bene o servizio pubblico, poniamo $\mathrm{Q}_{\mathrm{c}}$, mentre con la soluzione decentrata sarebbe fornito un output differente in ciascuna comunità. Dato il livello di output $\mathrm{Q}_{\mathrm{c}}$, la perdita di benessere del gruppo 1 è rappresentata nell'area $A B C$; mentre la perdita di benessere del gruppo 2 è rappresentata dall'area CED. Questo argomento, noto come teorema del decentramento, è stato formalizzato da Oates (Oates 1972) ${ }^{4}$.

${ }^{4}$ Un'altra giustificazione del decentramento è fondata sul criterio democratico: 
La superiorità dell'assetto decentrato non è più garantita secondo un criterio di benessere se si assume che le preferenze all'interno dei due gruppi non siano omogenee. Tuttavia, se si consente la mobilità degli individui, si può assicurare che le varie giurisdizioni corrispondano a gruppi con preferenze simili. In un modello divenuto classico, Tiebout (1956) cerca di determinare un'allocazione Pareto-ottimale dei beni pubblici attraverso l'associazione volontaria di individui che hanno gusti simili. Una delle conclusioni fondamentali della teoria economica è che le caratteristiche dei beni pubblici - non rivalità, non escludibilità - impediscono che il mercato generi un ottimo allocativo. Fare in modo che le persone rivelino le loro vere preferenze nel caso dei beni pubblici è reso problematico dalla possibilità del free riding. L'ipotesi di Tiebout è quella di costruire giurisdizioni in competizione che consentano alle persone di rivelare le loro preferenze «votando con le gambe». In questo modo le persone scelgono tra le giurisdizioni che forniscono la combinazione preferita di servizi pubblici e prelievo fiscale, raggruppandosi in comunità omogenee. Il modello si basa su alcune ipotesi estremamente restrittive quali la completa mobilità dei cittadini; la piena conoscenza delle caratteristiche delle varie giurisdizioni o comunità politiche; la disponibilità di un insieme di opzioni che coprano tutte le possibili combinazioni di beni e servizi pubblici desiderati dai cittadini; l'assenza di vincoli (familiari, geografici etc.). In altre parole, si tratta di un modello in cui la mobilità è senza costi. Nonostante la rigidità delle assunzioni, alla base di numerose critiche, l'accento posto sull'efficienza indotta dalla competizione tra unità governative resta un importante risultato della teoria economica, che ha generato anche una vasta letteratura empirica (Mueller 1989).

cfr. Pennock (1959) e Tullock (1969). Supponiamo che una unità governativa prenda le sue decisioni mediante la regola di maggioranza. Una maggioranza di cittadini preferisce la politica $\mathrm{A}$ alla politica $\mathrm{B}$. Il governo quindi realizza la politica $\mathrm{A}$ che favorisce la maggioranza e penalizza la minoranza. Supponiamo che l'area sia divisa in due unità, e ciascuna di esse voti su quella determinata questione. Se si realizza una maggioranza a favore di $\mathrm{A}$ in entrambe le unità, non si verifica alcun mutamento nella soddisfazione dei cittadini. Se tuttavia in una delle due nuove unità si realizza una maggioranza a favore di $\mathrm{B}$, il numero delle persone che vedono soddisfatte le loro preferenze aumenta necessariamente. Ad esempio, supponiamo che l'unità originaria abbia una popolazione di 1000 votanti, di cui 600 favoriscono A e 400 favoriscono B; ipotizziamo inoltre che in una delle due unità $\mathrm{B}$ sia preferito da una maggioranza di 300 persone; nell'altra $\mathrm{A}$ sia preferito da una maggioranza di 400 persone. Prima della suddivisione erano soddisfatte le preferenze di 600 votanti; dopo la suddivisione il loro numero è aumentato di 100 unità. 
Una volta individuate le ragioni che possono essere addotte per giustificare la creazione di una pluralità di livelli di governo, l'analisi economica si è tipicamente concentrata sul problema della determinazione di regole generali per l'assegnazione delle principali attività a differenti giurisdizioni (unità di governo responsabili di determinate funzioni economiche) e per il finanziamento di tali unità periferiches. Il problema della determinazione della dimensione delle giurisdizioni e quindi del loro numero è affrontato mediante l'applicazione del principio della perfetta corrispondenza tra l'area entro la quale ricadono i benefici dei servizi forniti dagli enti e i confini geografici della giurisdizione (Olson 1969; Oates 1972) ${ }^{6}$. Il risultato è un assetto decentrato caratterizzato da tanti livelli di governo quante sono le funzioni (i beni e i servizi pubblici) ripartite sul territorio in un numero determinato dalla loro dimensione ottima. $\mathrm{Si}$ tratta evidentemente di un principio formulato a livello normativo e quindi profondamente irrealistico. Nella realtà $\mathrm{i}$ confini geografici sono spesso storicamente dati; in virtù di questo, esistono effetti di spill over o di ricaduta esterna dei costi e dei benefici; esistono inoltre economie di scala nella produzione che agiscono da vincolo a un completo decentramento e giustificano l'esistenza o l'intervento di livelli di governo superiori.

Pur essendo fondamentalmente di ispirazione normativa, l'approccio economico offre un contributo importante all'analisi descrittiva o positiva dei sistemi federali, sottolineando che il decentramento politico consente l'emergenza di condizioni quasi competitive nell'offerta di beni e servizi pubblici. Ciò permette di migliorare la responsiveness del governo alle preferenze dei cittadini e l'efficienza nell'allocazione delle risorse. Questo risultato ha generato infinite variazioni sul tema, non soltanto all'interno dell'approccio economico (Breton 1987; Salmon 1987; Kincaid e Kenyon 1991; Wildawsky 1990; Dye 1990).

${ }^{5}$ La complessità di tali interazioni è oggetto di quell'insieme di contributi teorici che va sotto il nome di «federalismo fiscale» (Brown e Jackson 1990). Una rassegna esauriente di questa letteratura, oggi in una nuova fase di espansione (Stevens 1993), che pure rappresenta un contributo importante all'analisi dei sistemi federali (l'andamento delle relazioni finanziarie viene spesso assunto come indicatore dell'effettivo grado di decentramento) esula dagli intenti di questo lavoro.

${ }^{6}$ Un'altra strada per la determinazione dell'ottima struttura delle giurisdizioni è fornita dalla teoria dei club (Buchanan 1965). Questo modello fissa l'ampiezza ottimale della giurisdizione, in termini di membership, nel punto in cui il costo medio di fornire il bene pubblico locale è uguale al costo marginale derivante dall'aggiunta di un nuovo membro. 
Una delle critiche ricorrenti alla prospettiva economica strettamente intesa è che essa manca tipicamente di una teoria della politica. Focalizzandosi esclusivamente su problemi di scala o di output, essa assume le strutture istituzionali come date. Come osservano Brennan e Buchanan (1980) nella teoria convenzionale le varie funzioni governative sono assegnate ai differenti livelli di governo a seconda delle proprietà spaziali dei beni pubblici. La teoria stabilisce la soglia verso il basso dell'ampiezza della giurisdizione a cui dovrebbe essere assegnato il potere di finanziare e di fornire un certo bene o servizio (quella oltre la quale la frammentazione in unità più piccole genererebbe esternalità), ma non fa alcuna distinzione tra una struttura politica federale e uno stato unitario che decida di delegare alcune funzioni.

La public choice e la letteratura da essa derivata, nota anche come constitutional political economy, ha cercato parzialmente di colmare questa lacuna. Una critica all'approccio convenzionale in merito al problema del disegno dei livelli di governo è stata formulata da Breton e Scott (1978). L'analisi economica basata sulla internalizzazione dei costi e dei benefici conduce ipoteticamente alla frammentazione delle unità di governo, dal momento che non considera i costi di organizzazione. Breton e Scott suggeriscono che l'assegnazione delle diverse funzioni ai vari livelli di governo debba bilanciare vari tipi di costi, tra $\mathrm{i}$ quali i costi decisionali, che aumentano con la centralizzazione; i costi di coordinazione che, al contrario, aumentano con l'aumentare del numero delle giurisdizioni e, dal lato della doman$\mathrm{da}$, i costi di segnalazione delle preferenze e di partecipazione da parte dei cittadini.

Una critica più radicale è stata avanzata da Brennan e Buchanan (1980). L'approccio convenzionale adotta implicitamente una determinata concezione dello stato. Lo stato è trattato come un massimizzatore del benessere sociale, secondo un'assunzione che sta alla base anche dell'analisi economica dello stato unitario. L'approccio economico può essere concepito come un'analisi del governo «ideale», in quanto identifica le condizioni alle quali un governo motivato esclusivamente dall'interesse pubblico sceglierebbe di adottare differenti politiche a livello locale o anche di devolvere parte della sua autorità a unità di governo inferiori (Hamlin 1985). Mentre nella letteratura economica il federalismo è concepito come un mezzo per raggiungere politiche efficienti e desiderate dai cittadini di uno 
stato, la public choice pone l'accento sul federalismo come uno strumento per imporre vincoli alla discrezione governativa. In altri termini, nella conçezione emersa entro la public choice il federalismo si indirizza tipicamente alla soluzione di un problema di monopolio (Bish 1987).

Il principio che sta alla base del modello di Tiebout è la possibilità di impiegare l'uscita (il «voto con le gambe») come equivalente della voce ${ }^{7}$. Secondo Buchanan la disponibilità dell'opzione uscita va interpretata come uno strumento a disposizione dei cittadini-contribuenti per reagire alla tirannia del governo. Nei sistemi federali, in cui l'uscita è un'opzione a basso costo, la protezione dei cittadini è maggiore rispetto agli stati unitari, in cui esiste soltanto la possibilità della voce o in cui l'uscita presenta costi estremamente elevati. Pur essendo basati entrambi sulla competizione, $i$ due argomenti differiscono. L'assunzione implicita nell'approccio convenzionale è che il governo implementi criteri di efficienza. L'argomento di Buchanan si basa su un'assunzione motivazionale completamente diversa, e cioè che il governo debba essere modellato come un Leviatano interessato a massimizzare il surplus netto dati i vincoli esterni ed interni.

L'analisi più compiuta si trova in Brennan e Buchanan (1980) in cui il federalismo, inteso come dispersione dell'autorità fiscale tra unità governative in competizione, è presentato come l'equivalente di un vincolo costituzionale esplicito allo sfruttamento fiscale. In presenza di elevato accentramento e alti costi di mobilità i governanti perseguono attivamente assetti che favoriscono l'estrazione di rendite (Tullock 1969; Buchanan, Tollison e Tullock 1980). La competizione stimolata dalla disponibilità dell'opzione uscita diminuisce il potenziale di collusione e limita la capacità di sfruttamento fiscale dello stato: «la competizione intergovernativa per le risorse fiscali e la mobilità tra le diverse giurisdizioni degli individui che perseguono 'guadagni fiscali'

Come è noto, Hirschman (1970) traccia una distinzione tra processi in cui gli individui esprimono le loro preferenze attraverso l'esercizio dell'opzione uscita e quelli in cui la rivelazione delle preferenze avviene attraverso l'uso di qualche forma di comunicazione o di protesta. Tipicamente si assume che la politica sia caratterizzata dall'impossibilità del ricorso all'uscita: $\mathrm{i}$ cittadini possono astenersi dal partecipare, ma usualmente non è loro consentito lasciare il proprio paese per evitare decisioni sgradite. È interessante notare - come ha osservato lo stesso Hirschman (1993) - che exit e voice non sono necessariamente correlati in modo negativo. Per un'analisi del federalismo in questa chiave vedi ad esempio Eichenberger (1994). 
può offrire sostituti parziali o completi a espliciti vincoli fiscali sul potere di tassazione» (Brennan e Buchanan 1980, 184). Come ha osservato Hamlin (1985) la differenza tra i due modelli può essere formulata in termini di una semplice analogia. Il modello di Tiebout può essere paragonato a un certo numero di imprese che hanno «segmentato» con successo il mercato differenziando il loro prodotto; quello di Brennan e Buchanan richiede prezzi competitivi per prodotti identici. La differenziazione del prodotto introduce la possibilità che i governi locali sfruttino il loro potere di monopolio; una possibilità che secondo Brennan e Buchanan può essere ridotta solo aumentando il numero delle unità governative in competizione.

Il contributo di Brennan e Buchanan è importante perché collega l'analisi del federalismo alla scelta dei vincoli costituzionali. $\mathrm{E}$ in questa chiave che Buchanan interpreta anche la possibilità di inserire il diritto alla secessione nella costituzione degli stati federali ${ }^{8}$. Tuttavia, mentre il modello di Buchanan arricchisce l'analisi economica classica, continua a considerare lo stato come «produttore di beni pubblici, che è necessario vincolare in materie quali la massimizzazione del gettito fiscale per scopi 'impropri', o l'assegnazione 'inefficiente' della fornitura di beni pubblici a livello centrale» (Wiseman 1990, 118-119). In altri termini, non sviluppa compiutamente le implicazioni che derivano dal modellare lo stato come Leviatano, elemento che richiederebbe una considerazione più esplicita del ruolo di strutture e procedure come vincoli alla discrezionalità dell'azione governativa.

In sintesi, l'approccio economico e la public choice forniscono un complesso di argomenti relativi ai costi e ai benefici del decentramento politico. In tal modo essi si concentrano prevalentemente sull'analisi degli effetti del federalismo, inteso come sinonimo di decentramento sostanziale. Tali effetti consistono essenzialmente in un guadagno di efficienza indotto dalla creazione di una pluralità di unità governative in competizione. La public choice compie un passo ulteriore, collegando l'analisi del federalismo al problema dei vincoli costituzionali. Questo aspetto resta però solo parzialmente esplorato anche se alcuni

${ }^{8}$ Sul tema della secessione si è sviluppata una intensa riflessione, specie di tipo normativo: la posizione di Buchanan è articolata ad esempio in Buchanan e Faith (1987) e Buchanan (1991); un esempio molto interessante di analisi positiva è l'articolo di R.A. Young (1994) sul caso canadese. 
modelli e la letteratura da essi generata - in particolare la riflessione di Buchanan - pongono quanto meno l'esigenza di una prospettiva più ampia, che integri l'approccio economico strettamente inteso con l'analisi delle istituzioni politiche.

La teoria di Riker: la definizione del federalismo e la legge delle origini federali

La teoria di Riker è concepita in polemica con due filoni: la letteratura politico-giuridica tradizionale e l'approccio economico in senso stretto. Dal primo punto di vista, l'esigenza sottolineata da Riker è quella di fondare la teoria politica sulla costruzione di modelli deduttivi basati sull'assunto di razionalità degli attori; dal secondo punto di vista, quella di evidenziare i limiti dell'approccio economico - con cui condivide l'adesione a un comune apparato analitico - per quanto riguarda l'analisi delle istituzioni'. Per cogliere le differenze è importante esaminare sia la definizione di federalismo sia la teoria delle origini dei sistemi federali.

Come osserva lo stesso Riker in un contributo al manuale di Greenstein e Polsby che fa il punto degli studi sull'argomento fino al 1975, nella letteratura sono presenti varie definizioni del federalismo. L'approccio giuridico è esemplificato dalla definizione di Wheare (1964), secondo cui un governo è federale quando «riflette una divisione dei poteri tra autorità centrali e locali ciascuna delle quali è coordinata con le altre e indipendente da esse». Si tratta di un esempio di strategia «esclusiva», il cui ambito di applicabilità è ristretto ai sistemi federali classici (gli Stati Uniti, il Canada, l'Australia e la Svizzera). Già contestato da Livingston (1952) e da Friedrich (1968), l'approccio formalistico di Wheare viene scartato da Riker perché - oltre a

${ }^{9}$ In questa sede mi occupo soltanto della teoria descrittiva e non della valutazione normativa, o di quella che lo stesso Riker definisce l'«ideologia» del federalismo. Da quest'ultimo punto di vista, basti sottolineare che l'autore è molto critico nei confronti dell'equazione tra federalismo e libertà, sulla base del fatto che inizialmente il federalismo aveva consentito la protezione della schiavitù e della discriminazione razziale (è questo il senso dell'affermazione che chiude il volume del 1964: «chi è contro il razzismo negli Stati Uniti dev'essere contro il federalismo»). La riforma dei diritti civili del 1960 contribuisce a mutare questa posizione, e il federalismo viene giudicato non più un ostacolo al buon governo ma «un limite desiderabile, anche se non il più importante, al leviatano» (Riker 1987, XIII). 
escludere la Germania che tipicamente si caratterizza per lo svolgimento in comune di molte funzioni tra i Länder e il governo centrale - non si applica neanche al caso americano. Le ricerche di Grodzins (1966) e Elazar (1966) dimostrano infatti che la rigida separazione di funzioni presupposta dalla definizione di Wheare non esiste neppure nel caso americano. A questo proposito Grodzins ha coniato l'espressione «federalismo cooperativo», poi applicata soprattutto al caso tedesco ${ }^{10}$.

L'intento di Riker è quello di assicurare alla scienza politica una definizione operativa che rifiuti il linguaggio statico del diritto costituzionale: «il federalismo è un'organizzazione politica in cui le attività del governo sono divise tra governi locali e governo centrale in modo tale che ciascuno ha potere di decisione finale su alcune attività» (Riker 1975, 101). Tali sfere di attività possono essere più o meno numerose, fino al caso limite di una sola. Allo stesso modo Riker rifiuta di caratterizzare i sistemi federali in base a un elenco di funzioni o istituzioni caratteristiche - fissate da Wheare nella presenza di una costituzione scritta, di una procedura di revisione costituzionale che non sia rimessa esclusivamente al governo federale o al governo degli stati, di una Corte suprema che stabilisca il significato della costituzione in caso di controversie (questo elemento conduceva l'autore a considerare la Svizzera imperfettamente federale, in quanto priva di un'istituzione come la Corte suprema). La varietà istituzionale degli assetti federali maturi è tale infatti da sconsigliare il tentativo di procedere sulla base di rigidi elenchi di funzioni o di istituzioni caratteristiche, elemento che potrebbe precludere anche la considerazione degli aspetti dinamici ${ }^{11}$.

Se in questo modo Riker intende prendere le distanze dal formalismo dell'approccio giuridico, in un precedente lavoro offre una definizione che segna il distacco dall'approccio economico. Un sistema è federale se:

1) due livelli di governo hanno potere sullo stesso territorio e la stessa popolazione 2) ciascun livello ha almeno un'area in cui è autonomo 3) c'è qualche

${ }^{10}$ Sul federalismo tedesco si vedano ad es. Brecht (1967), Sharpf, Reissner e Schnabel (1976), Sharpf (1988).

"Su questo punto ha insistito anche Elazar (1987, 11-12): «L'essenza del federalismo non dev'essere ricercata in un particolare insieme di istituzioni, bensì nell'istituzionalizzazione di relazioni particolari tra i partecipanti alla vita politica. Di conseguenza, il federalismo è un fenomeno che fornisce opzioni diverse per l'organizzazione dell'autorità politica e del potere...». 
garanzia (anche un semplice pronunciamento della costituzione) dell'autonomia di ciascun livello di governo nella propria sfera (Riker 1964, 11).

L'elemento chiave della definizione riguarda le garanzie costituzionali dell'autonomia dei diversi livelli di governo. Ė questo ciò che distingue il federalismo dal semplice decentramento. $\mathrm{Su}$ questo aspetto Riker è tornato recentemente, definendo il federalismo come «governo articolato in più livelli costituzionalmente specificati» in esplicito disaccordo con i modelli economici. Tutti i governi sono organizzati in più livelli, ma nelle federazioni questo assetto organizzativo è riflesso in un accordo costituzionale permanente. «Se si ignora il fattore costituzionale [...] si trascura l'aspetto fondamentale del federalismo», ciò che lo distingue da un qualunque assetto decentrato: e cioè che «la struttura dei livelli di governo non è soggetta a revisione arbitraria» (Riker 1993, 509).

$\mathrm{Ne}$ deriva che il grado di accentramento/decentramento si può predicare tanto dei governi unitari quanto di quelli federali. La classificazione di Riker (1975 e 1993), che distingue in primo luogo tra unità indipendenti, alleanze, sistemi federali e unitari, può essere illustrata mediante la figura seguente:

$\begin{array}{ll}\text { max decentramento } & \text { max accentramento } \\ \text { unità indipendenti alleanze federazioni stati unitari } & \text { periferiche centralizzate }\end{array}$

A sua volta il federalismo può essere periferico (confederazione) o centralizzato (il federalismo americano è il primo esempio storico di federalismo centralizzato) ${ }^{12}$.

La definizione di Riker solleva alcuni problemi. L'individuazione della specificità del federalismo nelle garanzie costituzionali dell'autonomia dei diversi livelli di governo è generalmente condivisa. Sulla distinzione tra federalismo e decentramento concordano infatti numerosi altri autori, anche se la diversità dei termini impiegati può contribuire a generare confu-

$12 \mathrm{Da}$ questo punto di vista Riker si differenzia da quasi tutti gli altri che tendono invece a considerare quella tra confederazione e federazione una differenza di genus e non di species. Per una classificazione alternativa si veda ad esempio Elazar (1993). 
sione. Ad esempio Elazar preferisce indicare nel «non accentramento» la caratteristica distintiva del federalismo: «indipendentemente da come taluni poteri possano essere ripartiti tra il governo centrale e le unità componenti, l'autorità di partecipare all'esercizio di questi poteri non può essere ritirata senza il loro mutuo consenso» (Elazar 1987, 35-37). Al contrario, un sistema non federale implica l'esistenza di «una autorità centrale, di un governo centrale [...] che può decentrare o riaccentrare a suo piacimento...» (Elazar 1987, 34) ${ }^{13}$. Il modo migliore per sintetizzare questo punto è forse quello proposto da Martin Diamond (1969), che ha distinto tra federalismo contingente e federalismo costituzionale (cfr. anche Aranson 1990 e Ordeshook 1990). Il federalismo costituzionale differisce dal federalismo contingente in quanto esistono garanzie costituzionali relative alla sfera di autorità delle unità costitutive. Il governo centrale non può rovesciare unilateralmente tali garanzie e il processo di revisione costituzionale può avvenire soltanto attraverso procedure che prevedono il consenso delle unità periferiche. Nel federalismo contingente, è il governo centrale che decide quanta autorità delegare alle unità componenti e il grado di decentramento è quindi contingente o dipendente da queste scelte.

Non altrettanto condiviso è il fatto di ricomprendere nelle garanzie di autonomia dei livelli di governo inferiori il «semplice pronunciamento della costituzione». Riker è portato ad allargare la sfera dei referenti empirici anche a paesi solo formalmente federali - ad esempio l'Unione Sovietica prima del crollo o i paesi dell'America latina - pur considerando l'Unione Sovietica un esempio di federalismo massimamente accentrato in cui la garanzia di indipendenza delle unità costituenti sembra solo nominale. $\grave{E}$ anche su questa base che non ritiene necessaria la «clausola democratica», da altri considerata condizione essenziale per il funzionamento del federalismo ${ }^{14}$. Ostrom (1991) non accetta l'originaria formulazione di Riker ritenendo Riker.

${ }^{13}$ Su questo aspetto si veda anche Lijphart (1984), che accoglie la definizione di

${ }^{14}$ A questo proposito, non bisogna dimenticare che Riker si sforza di dare una definizione molto generale di federalismo. Per quanto riguarda i sistemi moderni, è vero che i casi menzionati non sono esempi di federazioni durevoli (Unione sovietica, Jugoslavia); d'altra parte, l'esempio della Nigeria mostra che l'assetto federale è sopravvissuto alle parentesi autoritarie in virtù del commitment delle élites politiche. Per quanto riguarda la Nigeria, si può addirittura affermare che il sistema federale sia stato utilizzato per favorire la transizione democratica: cfr. ad esempio Subaru (1994). 
troppo debole un semplice riferimento alla costituzione scritta e caratterizza il federalismo come «constitutional choice reiterated to apply to many units of government where each is bound by enforceable rules of constitutional law» (Ostrom 1987, 25). In altri termini, la costituzione dev'essere effettiva: il federalismo, come struttura di governo basata su molteplici centri di autorità, implica una enforceable rule of law. Vedremo nel paragrafo successivo come questa controversia possa avvantaggiarsi da un chiarimento sul significato della costituzione. Per il momento è sufficiente sottolineare che - nonostante Ostrom colga un punto importante - Riker pone con chiarezza il problema di che cosa mantiene il federalismo, quando osserva che i sistemi federali non possono basarsi unicamente sulla discrezione dell'autorità centrale, dato che l'incentivo a rovesciare la delega del potere è sempre presente.

Il distacco dall'analisi politica tradizionale e da quella economica si precisa anche nella spiegazione delle origini degli assetti federali. Riker intende pervenire a una teoria del federalismo esclusivamente basata su fattori politici. Il metodo seguito consiste in una procedura eliminativa volta a individuare le condizioni dotate di rilevanza causale e in un tentativo di incorporare le condizioni causali in un modello deduttivo. A questo proposito egli parla esplicitamente di una «legge delle origini federali», fondata sull'assunzione di razionalità degli attori. Le garanzie costituzionali che costituiscono la sostanza del federalismo sono infatti il risultato di un accordo volontario (constitutional bargain), che comporta vantaggi congiunti per i contraen$\mathrm{ti}^{15}$. Assumendo che gli attori politici tentino di stipulare accordi mutuamente vantaggiosi o Pareto-ottimali, ci si deve attendere un guadagno comune dalla creazione di assetti federali. L'obiettivo di Riker è esplicitare esattamente in che cosa esso consista.

Già nel volume del 1964 Riker individua nell'esistenza di una minaccia o di una opportunità di espansione territoriale la ragione fondamentale che spinge gli attori a stipulare il patto federale. Vengono stabilite così due condizioni del patto federale: 1) i politici che lo propongono desiderano espandere il loro controllo territoriale usualmente per far fronte a una minaccia esterna diplomatica o militare o in vista di una espansione territoriale, ma non sono in grado di farlo per incapacità militare. Il

\footnotetext{
15 All'elemento dell'accordo si riconduce la radice del termine, il latino foedus.
} 
federalismo è visto come un'alternativa all'annessione imperialistica, che comporta concessioni alle unità inferiori; 2) queste ultime, a loro volta, rinunciano a parte della loro indipendenza unendosi o perché desiderano protezione dalla minaccia o perché desiderano partecipare alla potenziale aggressione e considerano questi vantaggi superiori ai costi di rinunciare a parte della loro indipendenza.

L'ipotesi di Riker è che queste condizioni siano necessarie per la creazione di un assetto federale:

In ogni federazione che ha successo dev'essere presente una minaccia esterna o interna significativa o una significativa opportunità di aggressione, laddove la minaccia può essere fronteggiata e l'aggressione portata avanti solo con un governo più grande. Questo è ciò che [...] costituisce la «vincita» congiunta che giustifica l'accordo. Nello stesso tempo deve esistere qualche lealtà locale per rendere necessario l'accordo [...] ciò che previene la formazione di un governo unitario e introduce la federazione come un'alternativa (Riker 1975, 116).

Il carattere necessario di queste condizioni si rafforza se, per le federazioni non durevoli, si può mostrare che: 1) per quelle che si frammentano nelle unità costituenti, le minacce esterne o interne non sono significative; 2) per quelle che si trasformano in governi unitari, le lealtà locali sono relativamente deboli.

Riker è tornato su questa «legge» a distanza di vent'anni (Riker 1987). La salienza di queste condizioni è facilmente accertabile in federazioni che sono nate in seguito a una guerra o a una rivoluzione (la Svizzera, gli Stati Uniti), dal timore di una conquista (Canada), dall'anticipazione di un'aggressione (Australia). Ma, tra gli altri, Dikshit (1971) ha sostenuto ad esempio che mentre queste condizioni descrivono adeguatamente la nascita dei «vecchi» sistemi federali, in questo secolo le federazioni si sono formate per differenti ragioni, come è accaduto ad esempio nel caso della Germania occidentale e dell'India. Secondo Riker la Germania, che ha ereditato l'assetto federale del Reich imperiale (in cui costituiva uno strumento di espansione militare), ha adottato nuovamente il federalismo dopo la Seconda Guerra Mondiale nella prospettiva di una futura riunificazione con la Germania est. Analogamente, l'India nel 1948 ha adottato una costituzione federale in presenza di un conflitto territoriale col Pakistan. I vari casi di federazioni non durevoli sembrano conferire ulteriore sostegno all'ipotesi. L'unico autentico contro-esempio alla generalizzazione è fornito dalla Nige- 
ria, in cui la creazione del sistema federale non è stata accompagnata dalla presenza di una significativa minaccia esterna ma dalla prospettiva della guerra civile. Sulla base di questa critica, avanzata da Birch (1966), Riker riformula la generalizzazione tenendo conto della possibilità di una minaccia «interna» (Riker 1987).

A questa ipotesi Riker attribuisce un potere predittivo, asserendo esplicitamente che «tutte le future federazioni durevoli dovranno necessariamente essere accompagnate da queste condizioni». Queste ultime non sono infatti ricavate induttivamente, «ma dedotte dalla natura dell'accordo federale e delle istituzioni del federalismo»; a differenza dell'aspettativa di un vantaggio economico, che pure si trova frequentemente associata alla creazione di assetti federali, non vanno considerate «correlati importanti, ma fattori logicamente necessari». $\grave{E}$ questo il motivo per il quale ritiene che la scomparsa della minaccia sovietica allontani il compimento del processo di federazione europea e guarda con assoluto scetticismo all'idea di una federazione mondiale: «In assenza di una minaccia sufficientemente forte da rendere l'accordo federale reciprocamente vantaggioso per i governi che vi partecipano, non c'è niente che possa determinare unioni come queste» $(1975,127-131)$. Questa tesi viene riaffermata in uno scritto recente: «L'aggregazione delle risorse a scopi militari è il motivo primario, anche se strumentale, per la creazione di assetti federali» (Riker 1993, 510).

L'interpretazione «militare» del federalismo sembra tuttavia sfidata dai processi di federalizzazione in atto - il Belgio è il caso rilevante a questo proposito - $\mathrm{i}$ quali sono caratterizzati da una direzione inversa rispetto alle federazioni del passato: non sono cioè processi di integrazione ma di trasformazione pacifica di uno stato unitario in senso federale attraverso un processo di revisione della costituzione (Witte 1992). Riker (1993) spiega il processo di federalizzazione del Belgio o l'esportazione degli assetti federali in seguito ai processi di colonizzazione nei termini di un'altra condizione, cioè l'esistenza di un esempio altamente visibile di governo federale effettivo e praticabile, ovvero il federalismo americano. Il ricorso a questa condizione, altrove definita anch'essa «necessaria» o comunque altamente significativa (Riker 1975, 113), contrasta tuttavia con la perentorietà con cui viene affermata la rilevanza esclusiva delle due condizioni ricavate deduttivamente.

È possibile che la teoria di Riker sia valida per i processi di 
integrazione sovranazionale e che debba invece essere ripensata negli altri casi. Ritengo tuttavia che il problema sia più profondo, come mostrano le stesse perplessità dell'autore in merito alle conclusioni relative al destino politico dell'Europa (Riker afferma di considerare l'eventuale federazione dell'Europa un test di validità dell'ipotesi) ${ }^{16}$.

Soprattutto occorre ragionare sul fatto che non si tratta di condizioni «sufficienti», ma di condizioni «necessarie», dotate cioè di una forza esplicativa inferiore. Come riconosce Riker, non è possibile considerarle condizioni sufficienti (la minaccia sovietica non è bastata perché l'Europa si federasse; un altro esempio è costituito dalla dissoluzione della Jugoslavia, in cui, di fronte alla minaccia interna e in presenza di lealtà locali forti, l'esito ha coinciso con la guerra come alternativa alla soluzione negoziata). Né tantomeno si tratta di condizioni «necessarie e sufficienti», le sole che potrebbero conferire alla generalizzazione quel determinismo causale a cui Riker aspira e che - come lo stesso autore ha mostrato nei suoi saggi epistemologici e in altri importanti lavori ${ }^{17}$ - è possibile soltanto in presenza di un vero e proprio modello deduttivo. La mia impressione è che occorra distinguere tra la specificazione degli obiettivi nei termini del motivo più frequentemente associato alla nascita dei sistemi federali (cioè una questione empirica), e l'elaborazione di un modello basato sul calcolo razionale degli attori, necessariamente formulato a un superiore grado di astrazione. A questo proposito, l'analisi del processo di ratificazione della costituzione americana contribuisce già a mostrare come l'interpretazione «militare» si indebolisca alla luce del peso degli interessi economici, anche senza accettare la nota tesi di Charles Beard (1913/ 1935), che spiegava il sostegno o l'opposizione al nuovo sistema di governo esclusivamente sulla base di essi. Alcuni lavori recenti (McGuire e Ohsfeldt 1989; Eavey e Miller 1989) hanno tentato con successo di accertare empiricamente il peso esplicativo degli interessi economici in relazione a quelli legati alla percezione di una minaccia esterna, che pure si mostra fondamentale per spiegare il comportamento di alcuni stati chiave nel

${ }^{16}$ Sul destino politico dell'Europa nessun analista sembra disposto a fare previsio$\mathrm{ni}$, anche se si riconosce che l'evoluzione istituzionale europea presenta indubbiamente tratti federali (Sharpf 1988; Sbragia 1992).

${ }_{17}$ Per un approfondimento rimando alla mia Introduzione a Riker (1982), di prossima pubblicazione presso Edizioni di Comunità, Milano. 
processo di ratificazione. Se questa osservazione non intacca lo status di condizione «necessaria» attribuito agli scopi difensivi, sottolinea l'incompletezza della specificazione empirica degli obiettivi anche nel caso americano.

Il contributo forse più significativo della teoria di Riker all'analisi del federalismo riguarda il problema della stabilità degli assetti federali. Questo problema viene affrontato soprattutto in relazione al caso americano. La tesi di Riker è che il federalismo americano sia rimasto sostanzialmente immutato nel corso degli ultimi duecento anni dal punto di vista della stabilità politica e costituzionale, in netto contrasto con la tendenza all'accentramento amministrativo che lo stesso autore documenta. Che cosa ha reso le istituzioni del federalismo self-perpetuating? Riker $(1964 ; 1987)$ elenca quattro elementi della costituzione americana particolarmente rilevanti a questo proposito: a) il requisito che i legislatori risiedano negli stati che rappresentano; $b$ ) la flessibilità attribuita ai governi degli stati per quanto riguarda le modalità di elezione dei rappresentanti a livello nazionale; c) l'assenza di strumenti costituzionali (ad esempio il potere di sciogliere l'assemblea legislativa) che consenta ai presidenti di esercitare un controllo sulle nomine congressuali o sul partito di cui è espressione e il modo indiretto di elezione del presidente stesso. Combinati insieme, questi elementi producono una conseguenza ulteriore, che secondo Riker è condizione «sufficiente» della stabilità del sistema americano; $d$ ) due partiti decentrati, organizzati cioè a livello statale e locale. «Non la divisione formale dei poteri tra il governo centrale e i governi locali, ma il risultante decentramento dei partiti che rende impossibile la loro leadership a livello nazionale» impedisce la formazione di maggioranze che possano rovesciare la separazione costituzionale dei poteri (Riker 1982, 250). In altri termini, negli Stati Uniti non esistono due partiti nazionali, ma partiti che competono a livello statale e locale, anche se ogni quattro anni si coordinano per eleggere un presidente. I legislatori sono eletti secondo le regole stabilite dai vari stati e in seguito a campagne elettorali organizzate dai partiti su base locale; l'esistenza di un presidente che non può influenzare significativamente i loro destini elettorali fornisce un debole incentivo a formare forti organizzazioni a livello nazionale ${ }^{18}$. La conse-

${ }^{18}$ Questa tesi viene sostanzialmente accolta da Huntington (1968, 89): «Il solo ri- 
guenza è che l'assemblea legislativa protegge gli interessi degli stati. Allo stesso tempo, le coalizioni legislative non si formano strettamente su base locale grazie all'importanza della presidenza. L'aspirazione a controllare la presidenza fornisce ai partiti organizzati su base locale un incentivo a coalizzarsi a livello nazionale per eleggere il presidente. Il carattere bipartitico del sistema è dovuto alle regole elettorali, che disincentivano la formazione di terzi partiti. Il delicato equilibrio su cui si fonda il federalismo americano combina clausole costituzionali che decentrano le organizzazioni partitiche e contemporaneamente le spingono a negoziare $\mathrm{i}$ conflitti interni in modo da competere con successo per la presidenza. Questa spiegazione della stabilità del sistema americano, fondata esclusivamente su fattori istituzionali, rappresenta la principale alternativa alle ipotesi pluraliste che pongono l'accento sulle caratteristiche della società piuttosto che sul disegno delle istituzioni (Dahl 1994) ${ }^{19}$.

Nonostante gli elementi critici evidenziati in precedenza, la teoria di Riker è importante non solo perché rappresenta un tentativo rigoroso di inquadramento teorico, ma perché indirizza l'attenzione su due aspetti fondamentali. Il primo riguarda l'interpretazione delle origini del «patto» federale nei termini di un calcolo costi-benefici. Il secondo aspetto è relativo a quello che possiamo definire il problema della stabilità degli assetti federali, o di che cosa impedisce al governo centrale di rovesciare le garanzie di autonomia delle unità costituenti. All'analisi di questo problema offre un contributo importante la teoria politica positiva.

\section{Il federalismo nella teoria politica positiva}

Il programma di ricerca che va sotto il nome di neoistituzionalismo integra l'analisi economica e politica delle istituzioni all'interno di un medesimo apparato analitico che utilizza gli strumenti della teoria dei giochi, dell'economia dell'organizza-

vale potenziale al partito come istituzione distintiva della politica moderna è il federalismo».

19 Questa posizione è sintetizzata nella seguente affermazione di Dahl: «A mio parere, supporre che questo paese sia rimasto democratico grazie alla sua Costituzione significa chiaramente capovolgere la relazione reale; è molto più plausibile l'ipotesi che la Costituzione sia sopravvissuta perché è essenzialmente democratica» $(1994,163)$. 
zione e della teoria della scelta sociale. L'idea guida è che «le istituzioni contano» (Shepsle 1986; North 1990).

Questa prospettiva colloca l'analisi del federalismo nel contesto di una teoria positiva delle costituzioni, intese come insiemi di regole che governano il processo decisionale politico al livello più fondamentale. L'analisi prende le mosse dalla costituzione americana come prototipo delle costituzioni federali (Ostrom 1987; Grofman e Wittman 1989). L'elemento chiave è che la Costituzione americana, pur creando un governo abbastanza forte $\mathrm{da}$ garantire la difesa comune, i diritti di proprietà e il rispetto dei contratti, stabilisce nello stesso tempo un governo limitato.

La complessa struttura di governo creata con la costituzione americana - una pluralità di centri decisionali autonomi in concorrenza tra loro facenti riferimento a diverse e sovrapposte comunità di interesse - dipendeva criticamente dal rispetto dei limiti nell'esercizio delle prerogative governative, assicurato non solo dall'attribuzione dei diritti civili fondamentali (intesi come limiti all'autorità del governo) e dalla partecipazione popolare attraverso i meccanismi di rappresentanza, ma da un sistema costituzionale - fondato sulla separazione dei poteri - che sancisse la distinzione fondamentale tra legge costituzionale e legge ordinaria (Ostrom 1987). Madison tuttavia aveva ben chiaro che la semplice costituzione scritta non sarebbe stata sufficiente ad assicurare il rispetto dei limiti da essa stessa imposti:

Una semplice delimitazione sulla carta... non è garanzia sufficiente contro quelle violazioni che conducono a una concentrazione tirannica di tutti i poteri di governo nelle stesse mani (Federalist, n. 48).

Nel tentativo di comprendere «come funzionano i limiti costituzionali e perché alcuni di essi si dimostrano efficaci in pratica» (Weingast 1993, 287), la teoria politica positiva riconsidera il federalismo alla luce di due idee.

La prima, mutuata dalla riflessione condotta entro la teoria dei giochi, è che una costituzione debba essere interpretata come un meccanismo di coordinazione e non come un contratto (Hardin 1989). L'interpretazione della costituzione come un contratto sociale è tipica della public choice (Buchanan e Tullock 1962; Brennan e Buchanan 1985), secondo cui una costituzione rappresenta un insieme di regole per vincolare se stessi e le generazioni future a determinate azioni, ad esempio il rispetto di un patto fiscale. In questa prospettiva il contratto viene frequentemente interpretato come la soluzione a una singola in- 


\begin{tabular}{lcc}
\hline & & \\
& Cooperazione & Defezione \\
Cooperazione & 2,2 & 4,1 \\
Defezione & 1,4 & 3,3 \\
\hline
\end{tabular}

FIG. 2a. Gioco 1. Dilemma del prigioniero.

\begin{tabular}{lcc}
\hline & Cooperazione & Defezione \\
Cooperazione & 1,1 & 2,2 \\
Defezione & 3,3 & 1,1 \\
\hline
\end{tabular}

FIG. 2b. Gioco 2. Coordinazione.

\begin{tabular}{lcc}
\hline & Cooperazione & Defezione \\
Cooperazione & 2,1 & 3,3 \\
Defezione & 4,4 & 1,2 \\
\hline
\end{tabular}

FIG. 2c. Gioco 3. Coordinazione asimmetrica.

Fonte: Hardin (1989).

terazione modellata come un «dilemma del prigioniero» o un problema di scambio. Ciò che resta irrisolto in questo approccio è il problema dell'enforcement del contratto.

Per comprendere la differenza tra la struttura strategica di un «dilemma del prigioniero» e quella di un problema di coordinazione è utile confrontare le figure $2 \mathrm{a}, 2 \mathrm{~b}$ e $2 \mathrm{c}^{20}$.

La prima situazione descrive un tipico «dilemma del prigioniero», la cui «soluzione» richiede la creazione di incentivi, usualmente nella forma di sanzioni comminate da un terzo attore, per indurre gli attori a scegliere la cooperazione invece della defezione a cui li spinge la razionalità individuale. Nel secondo gioco, la scelta della cooperazione richiede semplicemente qualche tipo di segnalazione, per indurre i giocatori a scegliere le

20 I pagamenti sono espressi in forma ordinale, con 1 che corrisponde all'esito maggiormente preferito e 4 all'esito meno preferito. 
strategie I o le strategie II. Un gioco di coordinazione può presentare anche un certo grado di conflitto di interesse relativamente agli esiti: ad esempio, nel gioco 3 , un attore può preferire l'esito $(1,2)$ mentre l'altro può preferire $(2,1)$.

Una possibilità ulteriore è quella di concepire la costituzione come una soluzione a un «dilemma del prigioniero» iterato, analoga al processo di emergenza di una «convenzione» in seguito all'interazione ripetuta (contract by convention). Un risultato fondamentale della teoria dei giochi, noto come folk theorem, stabilisce che ogni interazione ripetuta è caratterizzata da una molteplicità di equilibri, alcuni dei quali asimmetrici o inefficienti (Fudenberg e Maskin 1986). L'implicazione fondamentale di questo teorema è che un equilibrio efficiente può essere raggiunto solo in presenza di un meccanismo di coordinazione - la comunicazione precedente, le strategie precedentemente usate o altri elementi «salienti» che caratterizzano il contesto strategico - che consenta ai giocatori di correlare le loro strategie in direzione di un esito mutuamente vantaggioso ${ }^{21}$. Come osserva Hardin, una costituzione non nasce da un insieme ripetuto di interazioni, anche se mostra analogie con questo processo. La creazione di una costituzione è piuttosto un atto singolo che regola un modello di interazioni future (Hardin 1989). Ad esempio, nel caso della Costituzione americana, i Padri fondatori avevano ben chiaro che il problema della difesa comune o del libero commercio costituivano questioni ricorrenti che, se lasciate irrisolte, avrebbero potuto condurre alla disintegrazione dell'Unione.

Concepire la costituzione come una «soluzione» a un problema di coordinazione implica una differenza cruciale rispetto all'approccio contrattualista. Mentre l'assimilazione della costituzione al contratto rimanda all'esistenza di un terzo attore che ne garantisca il rispetto (è questo il problema di regresso all'infinito che resta aperto nelle teorie contrattualiste), la costituzione intesa come meccanismo di coordinazione è self-enforcing, nel senso che corrisponde a un equilibrio che nessuno ha incentivo a mutare o perché Pareto-efficiente o a causa dei costi di coordinarsi nuovamente su un assetto alternativo. Questa reinterpretazione consente di comprendere il significato di una costituzione effettiva, come insieme di regole scritte o non scritte

${ }^{21}$ Il primo ad aver messo l'accento su questo punto è Schelling (1960). 
in grado di generare aspettative stabili circa il comportamento degli attori.

La seconda idea, mutuata dalla riflessione condotta entro la teoria della scelta sociale, è che gli esiti delle decisioni collettive sono influenzati non solo dalle preferenze ma anche dalle istituzioni, nel senso forte secondo cui a partire da preferenze identiche si possono produrre esiti diversi a seconda dei meccanismi decisionali impiegati (Riker 1982). Uno dei risultati più importanti della teoria della scelta sociale è costituito dall'analisi delle proprietà della regola di maggioranza. Questa riflessione ha riconosciuto da tempo che la regola della maggioranza - un elemento chiave della maggior parte dei sistemi democratici - ha anche proprietà indesiderabili. In particolare, data una opzione selezionata dalla maggioranza, ne esiste di solito un'altra, anch'essa preferita a maggioranza, che può rovesciare la prima. In altri termini, la regola di maggioranza è soggetta all'intransitività e al caos (Mc Kelvey 1976; Schofield 1978). L'instabilità può essere considerata una fonte di inefficienza, in quanto l'instabilità delle leggi significa instabilità dei diritti di proprietà: da qui l'incentivo a investire nel mutamento delle regole nel tentativo di assicurarsi vantaggi di breve periodo ${ }^{22}$.

Queste due idee sono state applicate allo studio dei sistemi federali - in particolare, gli Stati Uniti - sia per comprenderne la durata nel tempo sia per derivare regole o «principi» di disegno costituzionale di più ampia applicabilità.

Il primo tipo di analisi è oggetto dei lavori di Weingast $(1993,1995)$, che si concentra su un particolare sottoinsieme di sistemi federali, definiti market-preserving. Questi sistemi presentano una caratteristica aggiuntiva rispetto alle tre elencate da Riker, relativa ai limiti all'autorità di regolazione del governo centrale in materia economica e ai limiti al potere di erigere barriere protezionistiche da parte delle unità costituenti. Uno dei temi centrali del neoistituzionalismo è la spiegazione istituzionale delle variazioni nella performance delle varie economie nel tempo (North 1990). In particolare, lo sviluppo economico è spiegato in base alla presenza di istituzioni politiche che consentono ai governanti di stabilire «impegni credibili» in merito alla protezione di un sistema di diritti e garanzie. Il federalismo

${ }^{22}$ Come hanno mostrato Hardin (1989) e Hammond e Miller (1989), Madison era acutamente consapevole di questo aspetto. 
si è mostrato un fattore critico nello sviluppo economico di numerosi paesi - l'Olanda nel XVII secolo, gli Stati Uniti fino agli anni Trenta - creando condizioni competitive che hanno consentito di disincentivare i comportamenti collusivi e inefficienti. Tali effetti sono stati ampiamente studiati dalla public choice, mentre il problema scarsamente affrontato finora - con l'eccezione della teoria di Riker - riguarda la stabilità degli assetti federali stessi, o il modo in cui le istituzioni politiche hanno consentito ai governanti di porre vincoli efficaci alla propria autorità.

L'analisi di Weingast combina l'elaborazione di un modello in cui la scelta di rispettare i limiti costituzionali da parte del governo centrale e la reazione dei gruppi sociali a eventuali trasgressioni rappresentano possibili strategie in un gioco di coordinazione con la ricostruzione storica del federalismo americano, specialmente la fase cruciale che va dal 1828 al 1850. Quando le credenze degli individui o dei gruppi sociali rilevanti in merito agli appropriati limiti dello stato differiscono sensibilmente, la coordinazione è difficile e l'esito più naturale del gioco è un equilibrio asimmetrico (in cui i governanti trasgrediscono i limiti alla propria autorità coalizzandosi con alcuni gruppi contro altri). La costituzione può contribuire alla selezione di un equilibrio Pareto-efficiente, fornendo ai cittadini un modo simile di giudicare le azioni dello stato e rendendo possibile una reazione concertata a eventuali trasgressioni da parte del governo centrale. Interpretata alla luce di questo modello, l'analisi della storia americana mostra che il successo del federalismo poggia su una combinazione di vincoli formali (ad esempio la balance rule, o l'eguale rappresentanza degli Stati in Senato) e atteggiamenti o credenze circa l'appropriatezza di tali vincoli. Un adeguato insieme di istituzioni politiche è decisivo per mantenere un governo limitato: «istituzioni politiche adeguatamente specificate costituiscono lo strumento principale attraverso il quale i governi creano impegni credibili che vincolano la propria autorità» (Weingast 1993, 288; Williamson 1994). Tuttavia l'efficacia dei vincoli costituzionali dipende dall'emergere di un consenso circa gli appropriati limiti dello stato ${ }^{23}$.

${ }^{23}$ Si tratta di una reinterpretazione del concetto di legittimità nei termini della prospettiva basata sull'individualismo metodologico. Per una elaborazione di questo punto cfr. Iacobone (1995). 
L'elemento chiave è che questo modello tenta di incorporare nella spiegazione della stabilità variabili - come le credenze o gli atteggiamenti condivisi - finora escluse dall'analisi convenzionale, proponendosi di superare la dicotomia tra spiegazioni «economiche» e «culturali». Il punto da sottolineare è che istituzioni e credenze sono interpretate come proprietà di un equilibrio.

Il secondo tipo di analisi è stato affrontato da Ordeshook (1990 e 1992) in alcuni lavori che utilizzano i risultati della riflessione condotta nella teoria dei giochi e nella teoria della scelta sociale per derivare taluni criteri o principi di disegno costituzionale.

Il primo è che «il solo federalismo praticabile, se si intende avere una struttura federale, è quello costituzionale» (Ordeshook 1990). Il federalismo «contingente» non offre una protezione sufficiente dal rischio di abuso di autorità da parte del governo centrale. La costituzione tuttavia non dev'essere intesa nel senso di una semplice costituzione scritta, o solo nominale, ma come una costituzione effettiva che stabilisca un insieme di regole che inducono aspettative stabili. Nel linguaggio della teoria dei giochi, la costituzione deve corrispondere a un equilibrio nel gioco di coordinazione sottostante. In assenza di significative economie di scala o di una consistente minaccia militare, la coordinazione è difficile e ciò che si può ottenere è un trattato in cui differenti gruppi o entità si mettono d'accordo per cooperare su un insieme limitato di questioni. Il federalismo «periferico» che si fonda su semplici trattati di cooperazione (ad esempio, tra i paesi dell'ex Unione Sovietica) è l'unico possibile in società in cui esistono profonde divisioni etniche, linguistiche e religiose, intese come punti focali alternativi su cui si realizza la coordinazione. In generale, le costituzioni rappresentano soltanto uno dei meccanismi attraverso cui si realizza la coordinazione in una società; questo elemento va tenuto presente nella fase della loro progettazione. L'interpretazione in termini di coordinazione spiega anche perché, nel caso di una costituzione scritta, siano importanti i dettagli della formulazione e il dibattito pubblico in materia.

Il secondo principio è che la democrazia da sola non basta. Come dimostrano i risultati della teoria della scelta sociale, una maggioranza può sempre rovesciare un'altra maggioranza. Se, come afferma Riker (1980), le regole «ereditano l'instabilità degli esiti», che cosa impedisce che si formi una maggioranza in 
grado di rovesciare l'assetto costituzionale? Devono esistere istituzioni che vincolano la regola di maggioranza quali ad esempio il bicameralismo o la separazione dei poteri (Riker 1992; Hammond e Miller 1987 e 1989). Il federalismo stesso può essere interpretato come un meccanismo di stabilizzazione: la costituzione americana assegna sfere di competenza parzialmente sovrapposte a diverse giurisdizioni e attribuisce un potere di veto a ciascuna di esse nella propria sfera. Il sistema di giurisdizioni costituzionalmente definite può indurre la stabilità se si immagina che ciascuna issue locale possa essere interpretata come una dimensione in uno spazio $n$-dimensionale. Lo spazio a $n$-dimensioni genera instabilità, ma assegnando le issues alle giurisdizioni il federalismo induce un equilibrio che altrimenti non esisterebbe (Shepsle e Weingast 1981; Ordeshook 1990).

Negli Stati Uniti, il federalismo è parte di un sistema costituzionale più ampio, basato sul bicameralismo e la separazione dei poteri. Tutti questi meccanismi sono interpretabili come restrizioni alla sovranità della maggioranza, uno dei temi fondamentali del Federalist.

Come hanno mostrato Hammond e Miller, la ricerca della stabilità politica era uno dei principali obiettivi dei padri fondatori della costituzione americana: Madison difendeva la creazione del Senato nel quadro di un assetto bicamerale proprio su questa base. Il bicameralismo genera stabilità - cioè interrompe la ciclicità della regola di maggioranza - richiedendo che non tutte le coalizioni di maggioranza ma soltanto alcune siano decisive. Il bicameralismo non crea necessariamente la stabilità, qualora in entrambe le camere si realizzino le stesse maggioranze; la possibilità di indurre la stabilità dipende criticamente dall'esistenza di preferenze diverse in ciascuna camera. Come sottolineava già Madison, occorreva indurre nei senatori preferenze diverse da quelle dei deputati (modificando il metodo di elezione, i termini del mandato etc). In altri termini, il bicameralismo dev'essere differenziato.

Il bicameralismo è stato interpretato come un equivalente della regola di maggioranza qualificata, dal momento che eleva la quota di voti necessaria per l'approvazione di un determinato provvedimento (Buchanan e Tullock 1962). La separazione dei poteri non si limita ad elevare la quota di voti necessaria, ma induce una competizione tra i diversi poteri. Ciò richiede, in primo luogo, almeno tre unità decisionali caratterizzate da preferenze (incentivi) diversi; in secondo luogo regole che definiscano come 
un sottoinsieme di decisori può contrastare il terzo. Nella sua specificazione delle funzioni, la costituzione americana definisce almeno tre arene separate: la prima tra le tre istituzioni che interpretano la costituzione (la Corte suprema, il Presidente, il Senato); l'altra fra l'esecutivo e le due camere che formulano le leggi; la terza tra le due camere federali e le assemblee degli stati a cui è affidata la revisione della costituzione.

Perché si abbia competizione istituzionale occorre fare in modo che ciascuna abbia preferenze diverse, e ciò si può assicurare garantendo una diversa connessione con la sovranità, cioè incentivi elettorali che creino differenti preferenze (ad esempio, tra il Presidente e il Congresso).

Infine, la sopravvivenza del federalismo dipende criticamente da un'altra condizione, la cui importanza è stata sottolineata per primo da Riker, cioè il decentramento dei partiti. Ciò significa che le istituzioni rappresentative di un sistema federale devono essere disegnate in modo tale che i partiti politici nazionali mantengano una forte connessione con le costituencies locali, ad esempio attraverso il requisito che i legislatori risiedano nel collegio che rappresentano, o evitando sistemi elettorali proporzionali in cui $\mathrm{i}$ legislatori sono eletti senza avere rapporto con collegi locali. Nella progettazione del sistema elettorale, occorre bilanciare questo elemento con i rischi di frammentazione del sistema partitico: sono pertanto necessari incentivi elettorali che inducano le elítes partitiche locali a coalizzarsi a livello nazionale e a negoziare issues potenzialmente conflittuali entro la struttura partitica.

In sintesi, il problema affrontato entro la prospettiva neoistituzionalista è quello della stabilità costituzionale e istituzionale. Le conclusioni fondamentali che emergono da questa analisi possono essere riassunte nel modo seguente. Il federalismo può essere definito un «sistema praticabile di decentramento politico», intendendo con ciò la presenza di meccanismi istituzionali che rendono stabile la divisione dei livelli di governo. In altri termini, l'autonomia di ciascun livello di governo dev'essere istituzionalizzata in modo tale da rendere le restrizioni del federalismo self-enforcing. La combinazione dei vincoli formali può variare, ma alcuni meccanismi istituzionali piuttosto che altri - in particolare, quelli che impongono vincoli alla regola di maggioranza - sono maggiormente in grado di assicurare la stabilità costituzionale. Infine, il successo del federalismo dipende dall'emergere di un ampio consenso in merito al governo limitato. 


\section{Conclusioni}

In questo lavoro ho esaminato le tre principali direzioni di analisi del federalismo sviluppatesi all'interno dell'approccio razionale: quella che si può definire economica in senso stretto, quella politica elaborata nei numerosi lavori dedicati a questo tema da Riker, e quella neoistituzionalista. La prima offre un contributo indispensabile circa gli effetti del decentramento politico, in particolare le conseguenze in termini di efficienza indotte dalla competizione. Tuttavia fornisce un quadro incompleto, dato che non consente di cogliere la specificità istituzionale degli assetti federali. La seconda direzione di analisi fissa le linee generali di una teoria politica del federalismo concentrando l'attenzione sull'accordo costituzionale tra attori motivati dall'auto-interesse e sulla stabilità degli assetti federali. La prospettiva neoistituzionalista accoglie $\mathrm{i}$ risultati della public choice relativamente alle conseguenze economiche del decentramento politico, ma estende l'analisi all'ambiente istituzionale focalizzando l'interesse sul problema dell'efficacia dei vincoli costituzionali. Questo problema viene affrontato utilizzando sia i risultati ormai consolidati della teoria della scelta sociale - che consentono di interpretare assetti istituzionali complessi come meccanismi di stabilizzazione della regola di maggioranza - sia le idee emerse recentemente nell'ambito della teoria dei giochi, quali l'interpretazione della costituzione come «soluzione» a un problema di coordinazione. Pur essendo formulata a un elevato grado di astrazione, questa analisi consente di cogliere il significato di una costituzione effettiva, non solo evitando i dilemmi irrisolti nelle teorie contrattualiste ma anche offrendo una strada per lo sviluppo di una teoria positiva dei sistemi federali.

Le conseguenze di questa ricognizione teorica dal punto di vista della generazione di criteri per valutare la desiderabilità e la praticabilità dei progetti di riforma costituzionale in senso federale sono molteplici. Mi limito a elencarne due, le più rilevanti per il caso italiano, caratterizzato - al pari di altri paesi europei - dall'emergere di movimenti politici che hanno posto con forza la questione del federalismo nell'agenda politica.

La prima è che occorre avere ben chiara la differenza tra decentramento e federalismo. Di conseguenza, se si intende giustificare l'introduzione del federalismo, occorreranno ragioni ulteriori rispetto ai benefici economici e politici della competizione (che valgono anche per il decentramento, ammesso che que- 
st'ultimo sia effettivo). Queste ragioni hanno essenzialmente a che fare con il federalismo come parte di un sistema di separazione dei poteri, ovvero come meccanismo escogitato in funzione antipopulista (uso il termine nel senso impiegato da Riker) e antimaggioritaria. Sembra essenziale, da questo punto di vista, l'analisi di Riker laddove sottolinea la necessaria riduzione del peso dei partiti a livello nazionale.

Per quanto riguarda la praticabilità dei progetti di riforma in senso federale, disponiamo di una teoria come quella di Riker che si mostra piuttosto scettica sulla genesi dei sistemi federali per ragioni diverse da quelle legate alla difesa o alla conquista. Anche ammettendo che questa teoria sia incompleta, come sembrano indicare alcuni esempi di federazioni contemporanee, essa ha il merito di sottolineare il problema della stabilità dell'assetto federale. La lezione più importante che si può trarre da questa analisi è che la sopravvivenza del federalismo dipende non solo da una delicata e complessa combinazione di fattori costituzionali (nel caso americano, i meccanismi di rappresentanza, il ruolo della presidenza e così via) ma dalla loro incidenza su fattori extra-costituzionali, quali la forma organizzativa dei partiti politici. Da qui la futilità di discutere isolatamente di proposte di riforma che riguardino un singolo aspetto della struttura istituzionale.

\section{Riferimenti bibliografici}

Albertini, M. (a cura di) (1979), Il federalismo, Bologna, Il Mulino.

Aranson, P. (1990), Federalism: The Reason of Rules, in «Cato Journal», 10, 1, pp. 17-38.

Beam, D.R., T.J. Conlan e D.B. Walker (1983), Federalism. The Challenge of Conflicting Theories and Practices, in A. Finifter, Political Science: The State of the Discipline, Washington, APSA, pp. 247-279.

Beard, C. (1913/1935), An Economic Interpretation of the Constitution of the United States, New York, MacMillan.

Birch, A.H. (1966), Approaches to the Study of Federalism, in «Political Studies», 14, pp. 15-33.

Bish, R.L. (1987), Federalism: a Market Economics Perspective, in «Cato Journal», 7, 2, pp. 377-396.

Brecht, A. (1967), American and German Federalism: Political Differences, in Wildawsky, A. (a cura di), cit., pp. 185-189.

Brennan, G. e J.M. Buchanan (1980), The Power to Tax. The 
Analytical Foundations of a Fiscal Constitution, Cambridge, Cambridge University Press.

- (1985), The Reason of Rules. Constitutional Political Economy, Cambridge, Cambridge University Press; trad. it. La ragione delle regole. Economia politica costituzionale, Milano, Franco Angeli, 1991.

Breton, A. (1987), Towards a Theory of Competitive Federalism, in «European Journal of Political Economy», 3, pp. 263-329.

Breton, A. e A. Scott (1978), The Economic Constitution of Federal States, Toronto, University of Toronto Press.

Brosio, G. (1994), Equilibri instabili. Politica ed economia nell'evoluzione dei sistemi federali, Torino, Bollati Boringhieri.

Brown, C.V. e P.M. Jackson (1990, 4a ed.), Public Sector Economics, Cambridge, MA, Basil Blackwell.

Buchanan, J.M. (1965), An Economic Theory of Clubs, in «Economica», 32, pp. 1-14.

- (1991), An American Perspective on Europe's Constitutional Opportunity, in «Cato Journal», 10, 3, pp. 619-630.

Buchanan, J.M. e G. Tullock (1962), The Calculus of Consent. Logical Foundations of Constitutional Democracy, Ann Arbor, University of Michigan Press.

Buchanan, J.M. e R.L. Faith (1987), Secession and the Limits of Taxation: Toward a Theory of Internal Exit, in «American Economic Review» LXXVII, 5, pp. 1023-1031.

Buchanan, J.M., R.D. Tollison e G. Tullock (a cura di) (1980), Toward a Theory of the Rent-Seeking Society, College Station, Texas A\&M Press.

Dahl, R.A. (1956), A Preface to Democratic Theory, Chicago, University of Chicago Press; trad. it. Prefazione alla teoria democratica, Milano, Edizioni di Comunità, 1994.

Diamond, M. (1969), On the Relationship of Federalism and Decentralization, in Elazar, D. et al. (a cura di), Cooperation and Conflict: Readings in American Federalism, Itasca, Ill., Peacock Publ., pp. 72-82.

Dikshit, R.D. (1971), Military Interpretations of Federal Constitutions: A Critique, in «The Journal of Politics», XXXIII, pp. 180-189.

Dye, T.R. (1990), American Federalism: Competition Among Governments, Lexington, MA, Lexington Books.

Eavey, C.L. e G.J. Miller (1989), Constitutional Conflict in State and Nation, in B. Grofman e D. Wittman, cit., pp. 205-219.

Eichenberger, D. (1994), The Benefits of Federalism and the Risks of Overcentralization, in «Kyklos», XLVII, 3, pp. 403-420.

Elazar, D.J. (1966), American Federalism: a View From the States, New York, Crowell.

- (1987), Exploring Federalism, Tuscaloosa, University of Alabama 
Press, trad it. di prossima pubblicazione presso Edizioni di Comunità, Milano.

- (1993), International and Comparative Federalism, in «PS: Political Science \& Politics», XXVI, 2, pp. 190-195.

Friedrich, C.J. (1968), Trends of Federalism in Theory and Practice, New York, Praeger.

Fudenberg, D. e E. Maskin (1986), Folk Theorem in Repeated Games with Discounting and Incomplete Information, in «Econometrica», LIV, pp. 533-554.

Giannetti, D. (1993), Il neoistituzionalismo in scienza politica: il contributo della teoria della scelta razionale, in «Rivista Italiana di Scienza Politica», XXIII, 1, pp. 153-183.

Grodzins, M. (1966), The American System, Chicago, Rand McNally.

Grofman, B. e D. Wittman (a cura di) (1989), The Federalist Papers and the New Institutionalism, New York, Agathon Press.

Gwartney, J.D. e R.E. Wagner (a cura di) (1988), Political Economy and Public Policy, Greenwich, JAI Press.

Hamlin, A.P. (1985), The Political Economy of Constitutional Federalism, in «Public Choice», XLVI, pp. 187-195.

Hammond, T.H. e G.J. Miller (1987), The Core of the Constitution, in «American Political Science Review», LXXXI, pp. 1155-1174.

- (1989), Stability and Efficiency in a Separation of Powers Constitutional System, in B. Grofman e D. Wittman, cit., pp. 85-99.

Hardin, R. (1989), Why a Constitution?, in B. Grofman e D. Wittman, cit., pp. 100-120.

Hirschman, A.O. (1970), Exit, voice and loyalty, Cambridge, MA, Harvard University Press; trad. it. Uscita, defezione, protesta, Milano, Bompiani, 1982.

- (1993), Exit, Voice and the Fate of the German Democratic Republic, in «World Politics», XLV, pp. 173-202.

Huntington, S.P. (1968), Political Order in Changing Societies, Yale, Yale University Press; trad. it. Ordinamento politico e mutamento sociale, Milano, Franco Angeli, 1975.

Iacobone, N. (1995), Riscoprire la legittimità, working paper, Politeia, Milano.

Knight, J. (1992), Institutions and social conflict, Cambridge, Cambridge University Press.

Lijphart, A. (1984), Democracies, Yale University Press; trad. it. Le democrazie contemporanee, Bologna, Il Mulino, 1988.

Livingston, W.A. (1952), A Note on the Nature of Federalism, in «Political Science Quaterly», LXVII, pp. 81-95, ristampato in Wildawsky, A. (a cura di), cit., pp. 33-50.

Kincaid, J. e D. Kenyon (a cura di) (1991), Competition Among States and Local Government, Washington, The Urban Institute Press.

Mc Guire, R.A. e R.L. Ohsfeldt (1989), Public Choice Analysis and the 
Ratification of the Constitution, in B. Grofman e D. Wittman, cit., pp. 175-204.

Mc Kelvey, R.D. (1976), Intransitivities in Multidimensional Voting Models and Some Implications for Agenda Control, in «Journal of Economic Theory», XII, pp. 472-482.

Mueller, D.C. (1989), Public Choice II, Cambridge, Cambridge University Press.

Musgrave, R.A. (1959), The Theory of Public Finance, New York, Mac Graw Hill.

North, D.C. (1990), Institutions, Institutional Change and Economic Performance, Cambridge, Cambridge University Press; trad. it. Istituzioni, cambiamento istituzionale, evoluzione dell'economia, Bologna, Il Mulino, 1994.

Oates, W. (1972), Fiscal Federalism, London, Harcourt \& Brace.

Olson, M. (1969), The Principle of «Fiscal Equivalence»: the Division of Responsibility among Different Levels of Government, in «American Economic Review», LIX, pp. 479-487.

Ordeshook, P.C. (1990), Some Rules of Constitutional Design, in «Social Philosophy and Policy», VII, pp. 198-232.

- (1992), Constitutional Stability, in «Constitutional Political Economy», III, pp. 137-175.

Ostrom, V. (1987, 2a ed.), The Political Theory of a Compound Republic. Designing the American Experiment, Lincoln, University of Nebraska Press.

- (1991), The Meaning of American Federalism. Constituting a SelfGoverning Republic, San Francisco, ICS Press.

Pennock, R.J. (1959), Federal and Unitary Government: Disharmony and Frustration, in «Behavioral Science», IV, pp. 147-157.

Riker, W.H. (1964), Federalism: Origin, Operation, Significance, Boston, Little Brown.

- (1975), Federalism, in F. Greenstein e N. Polsby (a cura di), Handbook of Political Science, Reading, MA: Addison Wesley, pp. 93 172.

- (1980), Implications from the Disequilibrium of Majority Rule for the Study of Institutions, in «American Political Science Review», LXXVI, pp. 432-446.

- (1982), Liberalism against Populism. A Confrontation Between The Theory of Democracy and the Theory of Social Choice, San Francisco, Freeman, trad. it. di prossima pubblicazione presso Edizioni di Comunità, Milano.

- (1987), The Development of American Federalism, Boston, Kluwer.

- (1992), The Justification of Bicameralism, in «International Political Science Review», XIII, 1, pp. 101-116.

- (1993), Federalism in R. Goodin e P. Pettit (a cura di), A Compa- 
nion to Contemporary Political Philosophy, Cambridge, MA, Blackwell.

Salmon, P. (1987), Decentralisation as an Incentive Scheme, in «Oxford Review of Economic Policy», III, 2, pp. 24-43.

Sbragia, A. (a cura di) (1992), Euro-politics, Washington, The Brookings Institution.

Schofield, N. (1978), Instability of Simple Dynamic Games, in «The Review of Economic Studies», XLV, pp. 575-594.

Sharpf, F.W. (1988), The Joint-Decision Trap: Lessons from German Federalism and European Integration, in «Public Administration Review», LXVI, pp. 239-278.

Sharpf, F.W., B. Reissner e F. Schnabel (1976), Politikerverflechtung: Theorie und Empirie des kooperativen Federalismus in Bundesrepublik, Kronberg, Scriptor Verlag.

Shepsle K.A. (1986), Institutional Equilibrium and Equilibrium Institutions, in H. Weisberg (a cura di), Political Science: The Science of Politics, New York, Agathon Press, pp. 51-81.

Shepsle K.A. e B. Weingast (1981), Structure-induced Equilibrium and Legislative Choice, in «Public Choice», XXXVII, pp. 503-519.

Stevens, J.B. (1993), The Economics of Collective Choices, Westview, Boulder.

Subaru, R.T. (1994), The Travails of Federalism in Nigeria, in «Journal of Democracy», 4, 4, pp. 39-53.

Tiebout, C. (1956), A Pure Theory of Local Expenditures, in «Journal of Political Economy», 64, pp. 416-424.

Tullock, G. (1969), Federalism: Problems of Scale, in «Public Choice», 6, pp. 19-30.

Weingast, B.R. (1993), Constitutions and Governance Structures: The Political Foundations of Secure Markets, in «Journal of Institutional and Theoretical Economics», CXLIX, pp. 286-311.

- (1995), The Economic Role of Political Institutions: Market-Preserving Federalism and Economic Development, di prossima pubblicazione in «Journal of Law, Economics and Organization».

Wheare, K.C. (1964, 4a ed.), Federal Government, London, Oxford University Press.

Wildawsky, A. (1990), Federalism as Double Security: the Role of Competition, in «Cato Journal», X, 1, pp. 39-58.

Wildawsky, A. (a cura di) (1967), American Federalism in Perspective, Boston, Little Brown.

Williamson, O. (1994), Il ruolo della nuova economia istituzionale, in «Stato e Mercato», XL, pp. 41-68.

Wiseman, J. (1989), The Political Economy of Federalism: A Critical Appraisal in Cost, Choice and Political Economy, Aldershot, Edward Elgar, pp. 71-111.

- (1990), Principles of Political Economy. An Outline Proposal, Illu- 
strated by Application to Fiscal Federalism, in «Constitutional Political Economy», I, 1, pp. 101-124.

Witte, E. (1992), Belgian Federalism Towards Complexity and Asymmetry, in «West European Politics», XV, 4, pp. 95-117.

Young, R.A. (1994), The Political Economy of Secession: The Case of Quebec, in «Constitutional Political Economy», V, 2, pp. 221-245. 